\title{
RETRACTED ARTICLE: Effect of paclobutrazol \\ on photosynthesis and expression of pyrroline-5-carboxylatesynthase in contrasting wheat genotypes under water deficit stress condition
}

\author{
Sharad Kumar Dwivedi ${ }^{1}$ - Santosh Kumar ${ }^{1}$ \\ Received: 23 October 2015 / Accepted: 28 January 2016 / Published online: 9 February 2016 \\ (C) Prof. H.S. Srivastava Foundation for Science and Society 2016
}

The corresponding author retracts this article published at online first due to the unreliability of findings arising out of inappropriate handling of the photograph at the bottom right panel of Fig. 2. The authors regret the inappropriate image and thank the anonymous complainant at Pubpeer and the editor of PMBP for bringing it to their notice.

The online version of this article contains the full text of the retracted article as electronic supplementary material. 